\title{
La Leña como Metodología de Aprendizaje Activo para la Construcción
}

\author{
Arturo B. Rodriguez ${ }^{(1)}$, Camila Burgos Leiva ${ }^{(1)}$ y Washington Fernández ${ }^{(2)}$ \\ (1) Universidad Santiago de Chile, Departamento de Tecnologías Industriales, Grupo de Investigación en \\ Nuevas Tecnologías (GINT), Santiago, Chile. (e-mail: arturo.rodriguez@usach.cl; camila.burgos.l@usach.cl) \\ (2) Universidad del Bío-Bío, Departamento de Ingeniería Eléctrica, Concepción, Chile. \\ (e-mail:wfernand@ubiobio.cl)
}

Recibido Feb. 28, 2018; Aceptado May. 11, 2018; Versión final May. 29, 2018, Publicado Ago. 2018

\begin{abstract}
Resumen
El presente artículo muestra los resultados de una intervención del tipo activa utilizando tecnología electromagnética. La experiencia motivadora de la intervención logra ser centro de la atención de los estudiantes en la sala de clases. De esta forma el docente logra establecer una conexión desarrollando la comprensión de la humedad relativa en la leña. La intervención se realizó en dos semestres del año 2017, en la clase de materiales de construcción de la carrera Tecnología en Construcción. Las muestras fueron de similar género y grupo etario. Las variables medidas fueron cinco, las que determinan la comprensión de conceptos tales como tipificación, clasificación, reconocimiento y diferenciación. Los resultados obtenidos fueron significativos mostrando su enorme potencial como elemento de uso en la clase.
\end{abstract}

Palabras claves: electromagnetismo; comprensión; enseñanza-aprendizaje; docencia

\section{Firewood as an Active Learning Methodology for Construction}

\begin{abstract}
This article shows the results of an intervention of the active type using electromagnetic technology. The motivating experience of the intervention manages to be the center of attention of the students in the classroom. In this way the teacher can establish a connection by developing the understanding of the concept of relative humidity in firewood. The intervention was carried out in the two semesters of 2017, in the construction materials class of the technology career in construction. The samples were of similar gender and age group. The measured variables were five, which determine the understanding of concepts such as: typification, classification, recognition and differentiation. The results obtained were significant, showing its enormous potential as an element to be used in the class.
\end{abstract}

Keywords: electromagnetism; understanding; teaching-learning; teaching 


\section{INTRODUCCIÓN}

El avance tecnológico y su intervención en la sociedad es un tema de discusión desde hace ya algunos años y la necesidad de individuos y profesionales con capacidades tecnológicas es cada vez más solicitada. Sin embargo, esto contrasta con el fenómeno en el que las carreras profesionales del área de las ciencias, tecnología, ingeniería y matemáticas está sometida a una creciente deserción y a una disminución de la matrícula, que lleva a la comunidad educativa a reflexionar respecto de cómo abordar este tema. Estas áreas son conocidas por su acrónimo STEM (Science, Technology, Engineering y Mathematics), que es de mucha preocupación en Estados Unidos y Europa, donde los investigadores sostienen que las metodologías innovadoras y de actividades prácticas apoyarán la retención de estos estudiantes (Marin-Garcia et al., 2012; Lester et al., 2017). Es claro que el conocimiento y la información son elementos claves en el desarrollo tecnológico y que la educación debe estar inserta dentro de políticas nacionales y regionales que permitan un armonioso desarrollo con las actividades sociales (Knyazev et al., 2017). Otro tipo de metodología es el aprendizaje -servicio que logra vincular el aprendizaje y la responsabilidad social en el entorno, lo que permite un desarrollo cognitivo con sentido objetivo y de alcance social inmediato (Pulgar et al., 2014; Álvarez-Castillo et al., 2017).

Los estudiantes del ámbito STEM requieren cada día de una cobertura intensamente tecnológica al interior de la sala de clases, de lo contrario la califican la clase de aburrida. Hoy en el mundo la tendencia se ha instalado desde lo que se denomina Technology-Enhanced Learning (TEL) o Aprendizaje Mejorado en Tecnología (AMT), esto incluye conocimiento de hardware, dispositivos móviles, software, sistemas de gestión de aprendizaje y comunicación síncrona y asíncrona. Por lo general forma parte de programas profesionales y su aceptación está creciendo y sin duda se extrapolará a los programas de postgrado. Esta metodología persigue ser flexible, atractiva y orientada al estudiante basado en una alta interactividad, desarrolla autonomía e independencia del centro de aprendizaje siendo una de sus mayores virtudes (Scott et al., 2017; Machado et al., 2018). Dado que los nuevos estudiantes, aquellos que pertenecen a una sociedad altamente tecnologizada, requieren de metodologías con un alto grado de tecnología, se realizó una intervención en sala con un experimento que involucre uso tecnológico, participación, cooperación y colaboración, utilizando metodologías activas para sostener y permitir una elevada focalización en el proceso y una disminución de la dispersión (Rodríguez et al., 2015). Las metodologías activas unidas a las CoP (Community of Practice) o comunidades de práctica que buscan desarrollar y aprovecha la cooperación y colaboración y las $\mathrm{m}$-Learning o aprendizaje utilizando tecnología móvil tienen un enorme potencial sobre todo para potenciar la inclusión en las salas de clases (Martínez-Gómez, 2009; Tight et al., 2016; Jourian et al., 2017).

Por otro lado, la educación tecnológica está orientada hacia el TVET (Technical and Vocational Education and Training) o Educación y Formación Técnica y Vocacional, sin embargo la gran parte de los docentes en estas áreas son especialistas en el ámbito técnico y no en el ámbito pedagógico (Dickson et al., 2017). También existen muchas intervenciones en metodologías activas, como las basadas en problemas o ABP donde se construye una intervención alrededor de la solución individual o cooperativa de un problema que este asociado al conocimiento a impartir (Fonseca et al.; Watfa et al., 2017; Casanoves et al., 2017). El proyecto utilizó la tecnología electromagnética para mostrar a los estudiantes la importancia de la humedad (HR) de la madera y/o LEÑA. Se intervino un curso de la asignatura materiales de construcción de la carrera Tecnología en Construcción durante todo el semestre con un proyecto de aplicación tecnológica al problema de la LEÑA HUMEDA (Linville, 2005; Fernández-Golfin Seco, 2003, INFOR, 1991). Esta innovación plantea establecer que las metodologías activas ayudan a la comprensión y sintoniza la teoría con la práctica en el proceso E-A (Enseñanza - Aprendizaje) activo; dentro de su variado contenido se encuentran el estudio de los diversos materiales de construcción, características y pruebas de resistencia; cuya comprensión supone un desafío para los estudiantes y el docente. Desde la madera como material de construcción y la leña como material combustible se pretende establecer la importancia de la Humedad de los materiales para la construcción, ya que el tema de la construcción y la contaminación está presente en los medios y de suma importancia en la vida daría de los estudiantes (Nutsch, 2000; Pérez-Galaz et al., 1979).

Otros esfuerzos se centran en mejorar los procesos de enseñanza-aprendizaje a través del análisis del rendimiento académico respecto del perfil de ingreso de los estudiantes, identificando carencias formativas en sus procesos formativos. Estos estudios se han realizado en las carreras de ingeniería donde se observa una gran preocupación por detectar los elementos que benefician e impiden el mejoramiento del rendimiento académico (Etxeberria et al., 2017). Algunos estudios, en los que donde intervienen solo los estudiantes, han mostrado su preocupación por la calidad de la docencia, es decir se observa la calidad docente a través de la satisfacción de los estudiantes, donde se percibe la marcada tendencia de los procesos centrando al estudiante en el proceso enseñanza-aprendizaje, sin embargo, el estudiante no es el único actor en su proceso de aprendizaje, y sin duda no lo es en el proceso enseñanza aprendizaje. Pero, la metodología utilizada en estos estudios es un avance importante en la intencionalidad de medir la calidad en la docencia (González-Arias et al., 2016). 
También existen estudios que muestran revisiones sobre la evaluación del desempeño, pero desde la gestión de recursos humanos. Estos estudios visibilizan las deficientes eficacias de los sistemas donde se invierten grandes recursos dejando de lado otros donde se podría obtener mejores resultados. La gestión de los recursos y de los esfuerzos de los sistemas tiene una componente de análisis previo de las necesidades que muchas veces no se realiza y el sistema funciona casi auto automáticamente generando en la mayoría de las veces una inversión ineficiente de los recursos. La relevancia de los estudios y sus conclusiones muestran que se debe reforzar la implementación de mejoras, dichas mejoras deben ser de naturaleza táctica y operativa para fortalecer su eficacia. Para ello se deberá invertir en análisis de los requerimientos previos en educación basados en el desarrollo de la comprensión de los estudiantes y la evolución hacia el hacer (Cancino et al., 2015).

Después de revisar la literatura existente, podemos deducir que la comunidad educativa y las investigaciones están centradas en el proceso enseñanza-aprendizaje y en el desarrollo del hacer; a diferencia de los procesos centrados en el docente y el comprender como era antiguamente. Esto genera un individuo orientado al desarrollo de soluciones basadas en el reemplazo, por ejemplo: falla una máquina se detecta el módulo que no funciona e inmediatamente se reemplaza. Esto es útil cuando se trata de procesos seriales, como los que incorporan (o utilizan) las actuales industrias. Sin embargo, cuando se trata de problemas donde varios módulos de un proceso no funcionan la solución reemplazo se vuelve económicamente inviable. La necesidad de comprender un proceso es mucho más importante que la necesidad de su funcionalidad operativa. Las necesidades de las instituciones educativas se ven amplificadas por las carencias intrínsecas de los estudiantes y que en su mayoría son muy variadas y multidimensionales, lo que dificulta el desarrollo de metodologías que se apliquen de manera homogénea. Por lo tanto, se deben privilegiar metodologías de índole dinámica y que se implementen de manera heterogénea es decir a diferentes tipos de estilos de aprendizaje y modelo cognitivo, además de ser capaz de desarrollar y mejorar la comprensión de los estudiantes y que se basen el en el hacer como elemento funcional.

La metodología pretende una internalización del conocimiento y un anclaje significativamente superior al alcanzado actualmente con metodologías convencionales. El equipo investigador está convencido que se debe recuperar el valor de la comprensión de los procesos por encima de la importancia actual de la funcionalidad del proceso. Utilizando la metodología convencional, los estudiantes logran observar la diferencia de los materiales; pero con la aplicación tecnológica participativa (Metodologías Activas) se logrará incrementar la comprensión sobre las características de los materiales. Este proyecto ambiciona con instalar un concepto donde la comprensión es el camino hacia el "Hacer", donde el tránsito por la comprensión les dará seguridad y confianza sobre el conocimiento y desarrollar una actividad eficaz y eficiente con gran posibilidad de adaptabilidad por el propio estudiante (Tight et al., 2016).

\section{METODOLOGÍA}

La comprensión de la HR humedad en la leña es de vital importancia en nuestro medio debido a los efectos nocivos que trae consigo la quema de este material. La combustión de leña húmeda desarrolla material particulado en la atmosfera y esto no es deseable en un escenario de alta contaminación. La importancia de desarrollar estudiantes con elevada comprensión de la madera como material de construcción, se basa en la necesidad cada vez mayor del uso de este material y de la potencial contaminación por la combustión. En los últimos tiempos hemos sido testigos de los incendios de extensas áreas de bosque y de poblaciones cuyas construcciones están basadas en la madera.

El proyecto implementó una metodología que genera un incremento de la comprensión de los materiales y sus características a través de clases donde se involucra a estudiantes y docentes utilizando activamente la tecnología como vía para alcanzar el conocimiento de los fenómenos asociados a la madera en su versión material de construcción y material de combustión, de esta forma se logra mantenerlos focalizados en el objetivo del programa de estudio. Las clases anteriores a la aplicación del proyecto, no tuvo resultados importantes generando problemas para las asignaturas posteriores. En estas clases se utilizaba metodologías basadas en exposiciones y apoyadas por diapositivas, la naturaleza plana (bidimensional) de las clases no genera comprensión de los materiales y no permite mantener focalizados a los estudiantes. Mientras que las metodologías activas donde intervienen de manera simultánea, el docente y el estudiante, se logra internalizar el conocimiento, logrando posteriormente, modificar o aplicarlo en otros eventos.

Para ello se estableció el estudio de un fenómeno electromagnético sobre un volumen de madera, la misma que tenía humedad previamente definida. Los estudiantes observan el fenómeno, cuando se manifiesta la pérdida de potencia del campo electromagnético al atravesar la madera y/o leña húmeda, está pérdida de potencia depende de la humedad relativa presente, por tal razón se podrá estimar humedad del material expuesto al campo. Las figuras 1 y 2 resumen el estudio. 

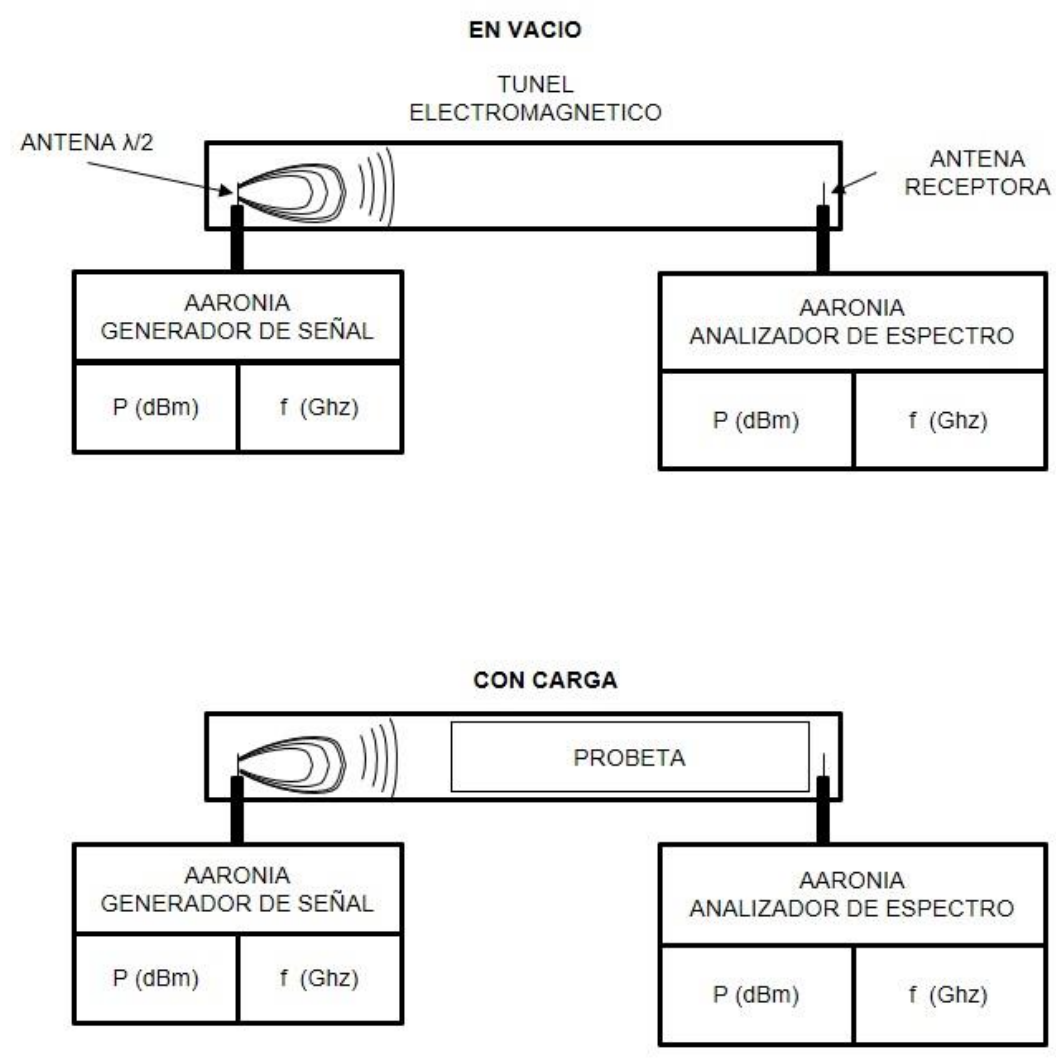

Fig. 1: Ensayo en Clase.

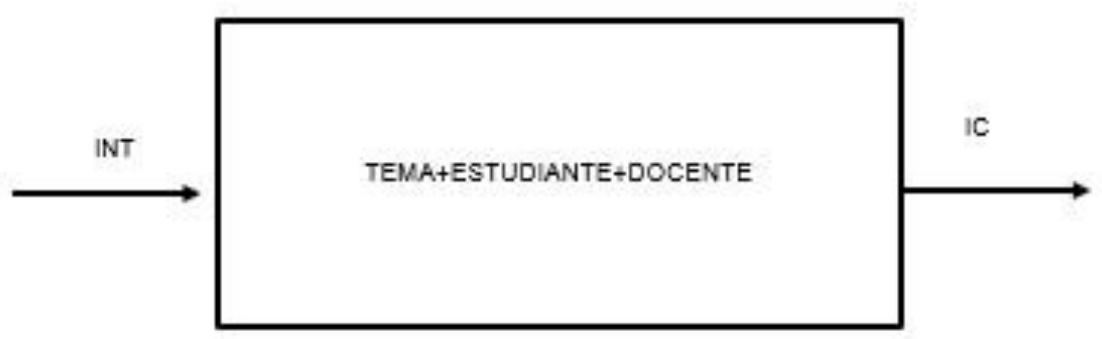

Fig. 2: Esquema de Intervención

Los ensayos se realizarán en diferentes grupos de la asignatura asociando diferentes niveles de humedad en el material. El estudio pretende establecer que la intervención causará una variación positiva en la comprensión del tema propuesto. Para este estudio se pueden destacar las variables independientes como el número de estudiantes y el tipo de intervención; y la variable dependiente que es el índice de comprensión similar al utilizado en (Rodríguez et al., 2017). Se evaluarán los Indicadores asociados a la comprensión, Índice de Comprensión Antes (ICA) e Índice de Comprensión Después (ICD) de las actividades de Implementación y además el Índice de Dispersión en Sala antes y después de la clase (IDA e IDD):

\section{Índice de Comprensión (IC)}

Este índice es utilizado para establecer las diferencias de lo comprendido en la sala de clases consta de un test de cinco preguntas determinadas por las variables Concepto (COO), Tipo (TIO), Diferencia (DIA), Clasificación ( $\mathrm{CLN}$ ) y Reconocimiento (RCO), en el ámbito de la temática materiales de construcción y humedad de la leña. El setting o escenario se establece desde la neutralidad y no hubo interacción verbal entre los protagonistas de la evaluación, la entrevista fue individual mediante tarjetas y dichas entrevistas fueron realizadas antes y después de cada clase donde. Los evaluadores fueron distintos y elegidos al azar de un set de 3 docentes. El instrumento de medicíon y las preguntas tuvieron 5 respuestas donde solo una fue correcta y el estudiante durante la entrevista solo debía señalarlo (Rodríguez et al., 2017). Una de las clases no ejecutó la intervención y tuvo una clase muy convencional con una clásica pizarra y el docente exponiendo; mientras que la otra clase se llevó a cabo con una metodología activa, cooperativa y colaborativa con la ejecución de un proyecto de investigación sobre la medición de la humedad con métodos convencionales y alternativos a través de radiactividad electromagnética. 


\section{RESULTADOS Y DISCUSIÓN}

La ejecución del proyecto fue en los semestres 2017-1 y 2017-2, las características muestrales de ambos grupos de estudio fueron de 19 años. Se puede observar el tamaño muestral en la tabla 1 y los grupos de estudio fueron del género masculino, por no tener en estos semestres estudiantes del género femenino. La metodología clásica en ausencia de intervención con metodología activa se realizó durante el semestre 20171 , donde un docente transmite y el estudiante recepciona, la que podemos llamar de metodología instructivista. Por otro lado, el semestre 2017-2 se realizó a la intervención metodológica donde los instrumentos de transmisión y medición de potencia electromagnética utilizado por el docente y la incorporación de la leña en el proceso, imprimió en la sala de clase una dinámica totalmente diferente y obteniendo la total atención de los estudiantes durante la clase.

Tabla 1: Muestra poblacional.

\begin{tabular}{|c|c|c|}
\hline & $2017-1$ & $2017-2$ \\
\hline Carrera & \multicolumn{2}{|c|}{ Tecnólogo en Construcción } \\
\hline Asignatura & Materiales de construcción & Materiales de construcción \\
\hline $\mathrm{N}$ & 15 & 16 \\
\hline
\end{tabular}

Para establecer cambios en la comprensión, se debió medir cinco componentes que permiten establecer con cierta cercanía dichos cambios, los mismos utilizados en (Rodríguez et al., 2017), en esta investigación se utilizó una rúbrica de cinco niveles donde 1 es la más baja y 5 es la más alta valoración. El IC (Índice de comprensión) se calculó como el aumento promedio de las variabilidades de cada dimensión.. La tabla 2, muestra el consolidado de respuestas promedio antes y después para los casos sin intervención (2017-1) y con intervención tecnológica (2017-2) donde se observa una gran diferencia en las respuestas para el caso de la clase con intervención tecnológica.

Tabla 2: Niveles de respuesta promedio según rúbrica por dimensión.

\begin{tabular}{|l|l|c|c|c|c|c|}
\hline & & COO & TIO & DIA & CLN & RCO \\
\hline $2017-1$ & Antes & 1,0 & 1,0 & 1,0 & 1,0 & 1,0 \\
\hline & Después & 3,0 & 2,0 & 2,0 & 3,0 & 1,0 \\
\hline $2017-2$ & Antes & 1,0 & 1,0 & 1,0 & 1,0 & 1,0 \\
\hline & Después & 5,0 & 4,0 & 4,0 & 3,5 & 4,5 \\
\hline
\end{tabular}

En la figura 3, se hace evidente la mejora respecto de la clase sin intervención tecnológica, donde existen diferencias significativas entre ambas metodologías, esto resultados se condicen con las recomendaciones y resultados que tienen de la aplicación del TEL (Technology Enhanced Learning) en el aprendizaje mostrado en (Scott et al., 2017). No cabe duda de que la intervención en la sala de clases es beneficiosa para el desarrollo cognitivo del estudiante, lo que podría permitir que los conocimientos obtenidos logren en el largo plazo, que los estudiantes prueben por ellos mismos y a su vez in situ el cambio de la variación de humedad en la leña. La tabla 3, muestra el porcentaje de estudiantes que responden correctamente después de cada clase donde se observa el aumento significativo de la comprensión. Es importante mencionar que la realización de experiencias durante las clases teóricas genera un ambiente de grato desarrollo tanto para el estudiante como para el profesor, generando mayor atención y disposición de los estudiantes frente a los contenidos.

En el trabajo de (Fonseca et al., 2017) muestra dos estrategias aplicando metodologías activad en dos universidades chilenas, donde se obtienen resultados similares y con un éxito similar, la diferencia se encuentra en la asignatura donde se aplicó la metodología, donde el trabajo referenciado lo aplicó en ingeniería de software que es una asignatura más teórica y menos práctica; mientras que el trabajo presentado fue aplicado en una asignatura más práctica y menos teórica. Sin embargo, ambos resultados se refuerzan indicando que la aplicación de tecnología en la sala de clases beneficia sin duda alguna al proceso enseñanza aprendizaje. Un tema no discutido en la literatura es el aporte de la tecnología a la disminución de la dispersión de los estudiantes en clase, logrando una mayor focalización de los estudiantes en el tema de discusión docente. Por otro lado, se observaron los cambios respecto de la respuesta correcta sin tomar en cuenta la evaluación por rúbrica. No olvidemos que la evaluación por rúbrica depende de la objetividad del evaluador, mientras que la respuesta depende exclusivamente del evaluado y por consecuencia estaría sujeta inclusive a la aleatoriedad. En la figura 4, se muestra las respuestas más frecuentes de los estudiantes antes y después de la intervención tecnológica, se hace visible el aporte de la metodología en las dimensiones de reconocimiento y diferenciación, ambas importantes en la comprensión temática de la leña como elemento de construcción. 


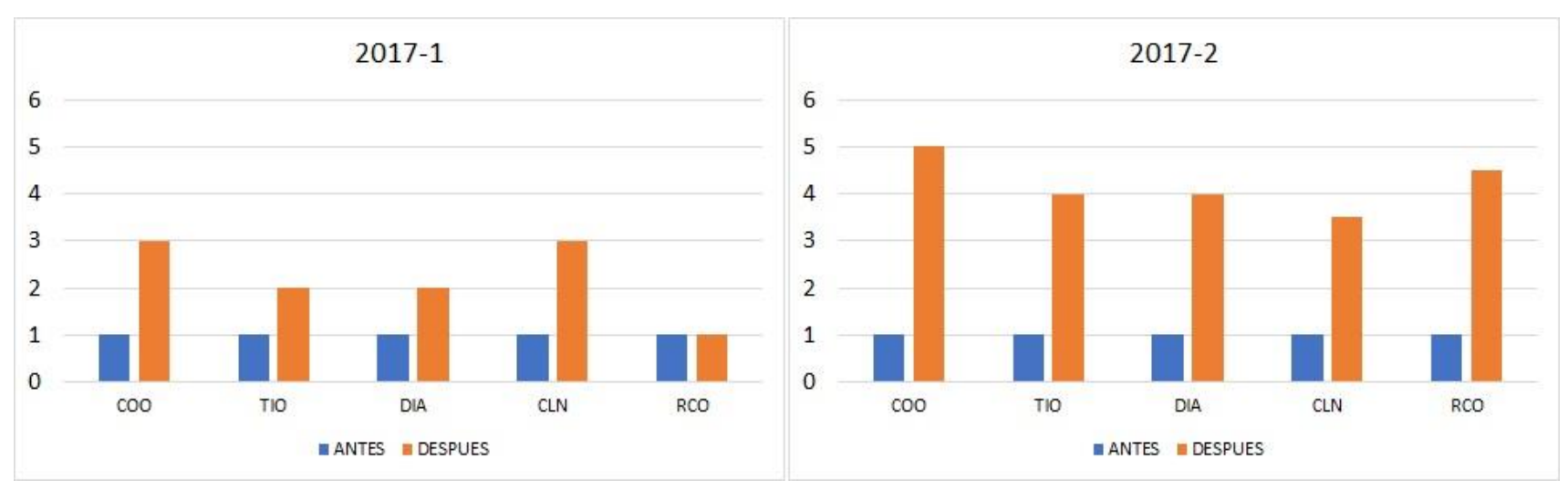

Fig. 3: Comparación de resultados sin y con intervención tecnológica

Tabla 3: Porcentaje de estudiantes que responden correctamente después de cada clase.

\begin{tabular}{|c|c|c|c|c|c|}
\hline & COO & TIO & DIA & CLN & RCO \\
\hline $2017-1$ & $30 \%$ & $32 \%$ & $42 \%$ & $38 \%$ & $45 \%$ \\
\hline $2017-2$ & $65 \%$ & $75 \%$ & $60 \%$ & $85 \%$ & $55 \%$ \\
\hline
\end{tabular}

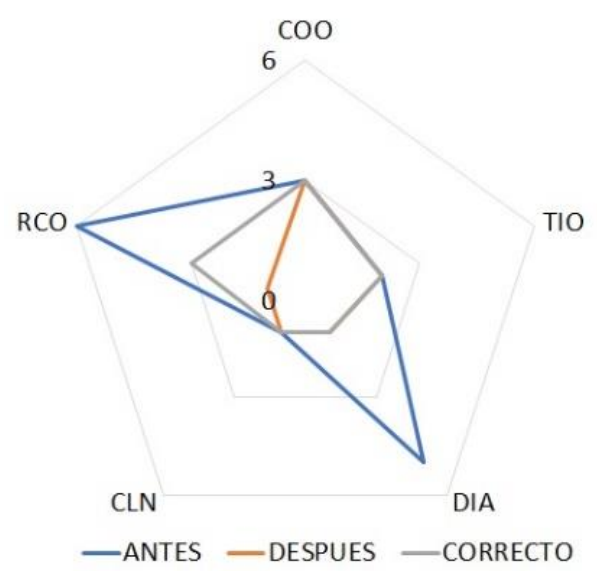

Fig. 4: Comparación de resultados antes y después de la intervención 2017-2

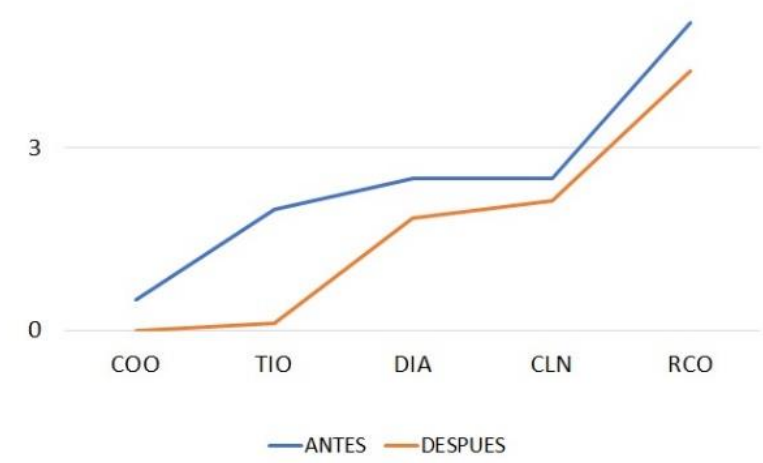

Fig. 5: Distribución de las varianzas respecto de las respuestas correctas

La figura 5, muestra como las respuestas de los estudiantes antes de la intervención varían de la respuesta correcta y luego disminuyen, nótese que una varianza cero indica conocimiento de la respuesta evidenciando conocimiento previo o aleatoriedad, en todo caso se observa que después de la intervención el grupo de estudio mejoró y se acercó más a la respuesta correcta, indicando mejoramiento de la comprensión, esto se observa por la disminución de la variabilidad promedio respecto de la respuesta correcta, utilizando las tarjetas de comprensión. 


\section{CONCLUSIONES}

La comprensión es una dimensión muy difícil de observar y mucho más de medir, debido a la gran espectro de acepciones que esta contiene, sin embargo, la metodología propuesta logra evidencias rasgos que permiten establecer si la comprensión aumentó o disminuyó o verificar la eficiencia de una metodología, en este caso se utilizó una metodología activa. Se observó una enorme mejora respecto de la atención de los estudiantes, y logrando una mejora en la dinámica de la clase con mucha partición de los estudiantes durante las clases.

Es importante ver la utilidad de las metodologías activas y su potencial uso en las clases sobre todo teóricas, donde la dispersión de la atención estudiantil es cada vez más evidente. La intervención mostró la mejora de los índices en aproximadamente $31 \%$ en promedio con un máximo de $47 \%$ en clasificación de la madera y un mínimo de $10 \%$ en reconocimiento, lo que indica su eficacia en el proceso enseñanza-aprendizaje. Por lo tanto, se espera su incorporación en los programas de los próximos semestres. Otro importante elemento es el logro de transversalizar las disciplinas y desarrollar sinergia entre las diferentes carreras que es lo que se logró en esta oportunidad.

\section{AGRADECIMIENTOS}

Para la Vicerrectoría Académica de la Universidad de Santiago de Chile - USACH (Proyecto PID № 0052015) y al Grupo de Investigación en Nuevas Tecnologías (GINT-DTI-USACH).

\section{REFERENCIAS}

Álvarez-Castillo, J., M. Martínez-Usarralde, H. González-González y M. Buenestado-Fernandez, Service-learning in teacher training in Spanish universities, Revista Española de Pedagogía, 75(267), 199-217 (2017)

Cancino, V. y T. Márquez, Evaluación de Desempeño de la Función Académica: Análisis de un Sistema en el Contexto Universitario Chileno, Formación Universitaria, 8(3), 35-46 (2015)

Casanoves, M., Z. Salvadó, Á. González, C. Valls y M. Novo, Learning genetics through a scientific inquiry game, Journal of Biological Education, 51(2), 99-106 (2017)

Dickson, M. y S. Ladefoged, Introducing active learning pedagogy into a technical and vocational education and training academy in Kurdistan, Iraq, International Review of Education, 63(5), 679-702 (2017)

Etxeberria, P., E. Alberdi, I. Eguia y Må . José García, Análisis del Rendimiento Académico en Relación al Perfil de Ingreso del Alumnado e Identificación de Carencias Formativas en Materias Básicas de dos Grados de Ingeniería, Formación Universitaria, 10(4), 67-74 (2017)

Fernández-Golfin, S., M. Díez, E. Hermoso y R. Amier, Manual de clasificación de madera, 1ª Ed.,12-24, Aitim, Madrid, España (2003)

Fonseca, V. y J. Gomez, Applying Active Methodologies for Teaching Software Engineering in Computer Engineering, Revista Iberoamericana de Tecnologias del Aprendizaje, 12(3), 147-155 (2017)

González-Arias, M., E. Carabantes y N. Muñoz-Carreño, Construcción y Validación de la Escala de Apreciación de la Calidad del Programa de Asignatura: Propuesta para el Estudio de la Calidad de la Docencia, Formación Universitaria, 9(1), 77-90 (2016)

Jourian, T. y Z. Nicolazzo, Bringing our communities to the research table: the liberatory potential of collaborative methodological practices alongside LGBTQ participants, Educational Action Research, 25(4), 594-609 (2017)

Knyazev, N., R. Buyankina y R. Zukov, The relationship between knowledge and information in the development of modern social practices, Novosibirsk State Pedagogical University Bulletin, 7(3), 124-139 (2017)

Lester, J. y A. Kezar, Strategies and Challenges for Distributing Leadership in Communities of Practice, Journal of Leadership Studies, 10(4), 17-34 (2017)

Linville, J., Timber Construction Manual, 6 a Ed., John Wiley \& Sons, 1-41, New Jersey, USA (2012)

Machado, L., J. da-Silva-Mendes, D. Sampaio, T. Grande y P. Behar, Smart Education and e-Learning, 1를., 391-399 Springer, Cham, Switzerland (2018)

Marin-Garcia, J. y A. Conchado-Peiró, Influencia de los Perfiles Docentes en el Uso y Preferencia de Dinámicas de Trabajo en Grupo con Alumnos Universitarios, Formación Universitaria, 5(5), 3-14 (2012)

Martínez-Gómez, M. y J. Marín - García, Como Medir y Guiar Cambios hacia Entornos Educativos más Motivadores, Formación Universitaria, 2(4), 3-14 (2009)

Nutsch, W., Tecnología de la madera y el mueble, 1르. Ed., 72-76, Editorial Reverté, Barcelona, España (2000)

Pérez-Galaz, V. y C. Alarcón, Sistemas constructivos en madera, Manual 12, $1^{\text {a }}$ Ed., 38-40, Instituto Forestal de Chile, Chile (1979) 
Pérez-Galaz, V., Manual de Construcciones en Madera, Manual 10, 2a Ed., 113-117, Centro de Transferencia Tecnológica INFOR, Santiago, Chile (1991)

Pulgar, J. e I. Sánchez, Impacto de un programa de renovación metodológica en las estrategias cognitivas y el rendimiento académico en cursos de física universitaria, Formación Universitaria, 7(5), 3-14 (2014)

Rodríguez A., L. Ramiréz y W. Fernandez, Metodologías Activas para alcanzar la comprensión, Formación Universitaria, 10(1), 79-88 (2017)

Scott, K., L. Baur y J. Barrett, Evidence-Based Principles for Using Technology-Enhanced Learning in the Continuing Professional Development of Health Professionals, Journal of Continuing Education in the Health Professions, 37(1), 6166 (2017)

Tight, M., Phenomenography: the development and application of an innovative research design in higher education research, International Journal Of Social Research Methodology, 19(3), 319-338 (2016)

Watfa, M. y D. Audi, Innovative virtual and collaborative teaching methodologies, Behaviour and Information Technology, 36(7), 663-673 (2017) 